What's New from

Juntendo University, Tokyo

Juntendo Medical Journal

2014. $60(6), 520-522$

\title{
Summary of 334th Triannual Meeting of the Juntendo Medical Society "Future Perspectives of Robotic Surgery in Juntendo"
}

\author{
TOSHIYUKI CHINA*1), TERUMASA MORITA*2), SHIAKI OH*3), \\ SHIN-ICHI OHBA*4), KIYOHITO NAITO*5), HIROYUKI KOGA*6), \\ YASUHISA TERAO*7), ATSUYUKI YAMATAKA*6), SHIGEO HORIE*1)
}

\begin{abstract}
*1) Department of Urology, Juntendo University Faculty of Medicine, Tokyo, Japan, *2) Department of Cardiovascular Surgery, Juntendo University Faculty of Medicine, Tokyo, Japan, *3) Department of General Thoracic Surgery, Juntendo University Faculty of Medicine, Tokyo, Japan, *4) Department of Otorhinolaryngology-Head and Neck Surgery, Juntendo University Faculty of Medicine, Tokyo, Japan, *5) Department of Orthopaedics, Juntendo University Faculty of Medicine, Tokyo, Japan, *6) Department of Pediatric General and Urogenital Surgery, Juntendo University Faculty of Medicine, Tokyo,

Japan, *7) Department of Obstetrics and Gynecology, Juntendo University Faculty of Medicine, Tokyo, Japan
\end{abstract}

Aim: To present the current status and future perspectives of robotic surgery in individual subspecialties.

Scope: robotic surgery, surgical navigation, simulation, telesurgery

\section{Robot-assisted surgical navigation in urology (Toshiyuki China)}

In the 1980s, the development of robotic surgery was initiated in the US for telesurgery on the battlefield. In 1999, Intuitive Surgical Inc. announced the da Vinci system, which received approval from the FDA for general laparoscopic surgery in 2000. In Japan, radical prostatectomy for prostate cancer became covered under the national health insurance system in April 2012. At Juntendo Hospital, a new model of the da Vinci system, Si, was introduced in July 2013. Currently, more than 3,000 robotic surgical systems are in use worldwide, including over 2,000 in the US and over 180 in Japan. In the urology service at Juntendo Hospital, already close to 200 operations have been performed using da Vinci, including radical prostatectomy, partial nephrectomy, and radical cystectomy.

Partial nephrectomy requires correct extirpation of the tumor as well as maximum sparing of renal parenchyma. To achieve these goals, we have developed computer-assisted surgical navigation

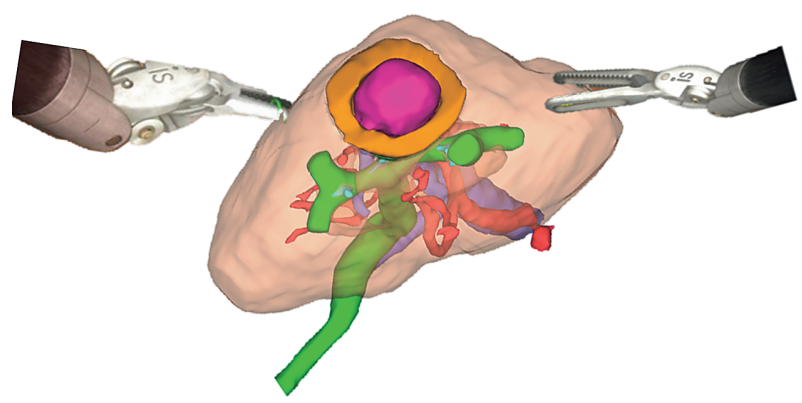

Figure-1 Incorporating our surgical planning image into da Vinci console

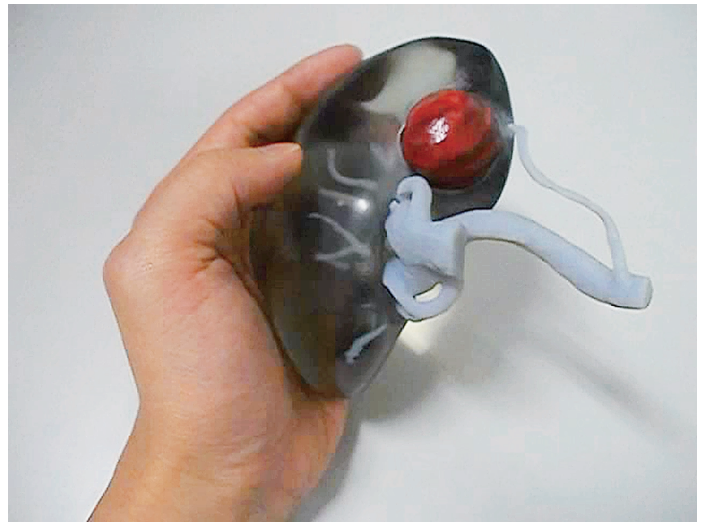

Figure-2 3D real-size kidney and tumor

Corresponding author: Shigeo Horie

Department of Urology, Juntendo University Faculty of Medicine

2-1-1 Hongo, Bunkyo-ku,Tokyo 113-8421, Japan

TEL: +81-3-3813-3111 E-mail: shorie@juntendo.ac.jp

[Received Dec. 9, 2014] 
(CASN). Our CASN is useful for identifying selective renal artery clumping and for evaluating post-operative renal function (Figure-1). By utilizing the TilePro function of da Vinci, we incorporated this image into the console view in order to achieve more precise surgical navigation. In da Vinci, surgeons lose their tactile sensation. This is a particular disadvantage in cancer surgery, where palpation of the organ or tissue can reveal the existence and boundary of the tumor. To overcome this drawback, we routinely utilize life-sized kidney and tumor models made using a 3D printer based on MDCT DICOM data (Figure-2).

\section{Simulation using 3D-CT imaging for robotic cardiac procedures (Terumasa Morita)}

In robotic cardiac procedures, the position of the endoscopic port is crucial for visualization of the heart and risk minimization of heart injury caused by the robotic arms. Thus, pre-operative assessment of port placement for the robot arm and the endoscope is extremely important to perform a high-quality procedure. 3D-CT imaging for mitral valve plasty using artificial chordae was demonstrated. In addition, the effective use of endoscope mode and opacity control may dramatically increase the safety of the procedure.

\section{Robotic thoracic surgery (Shiaki Oh)}

There are three operative approaches in thoracic surgery, which are open, thoracoscopic, and robotic. In Japan, open and thoracoscopic surgeries are approved, but robotic operations are not. Although the chest cavity is narrow, robotic operation is considered to be useful for lung cancer, mediastinal tumor, and thymic disease. However, at present, there is no literature showing that robotic surgery could improve the mortality rate and the length of hospital stay for these operations. There are some obstacles to the introduction of robotic thoracic surgery in Japan, such as technical difficulty, cost, and safety. By overcoming these issues, the benefits of robotic thoracic surgery should be demonstrated in the near future.

\section{Transoral robotic surgery (Shin-ichi Ohba)}

Transoral robotic surgery (TORS) has gained importance in the resection of head and neck tumors worldwide. Oropharyngeal cancer, especially $\mathrm{T} 1$ and $\mathrm{T} 2$ tumors of the tongue base and lateral wall, could be easily visualized with the help of the da Vinci system. The benefits of TORS over traditional open surgery include hand tremor reduction, better visualization, and minimization of surgical trauma. Furthermore, TORS could preserve better swallowing function over primary chemo-radiation or conventional open surgery. Our department will prepare for the introduction of TORS before its pharmaceutical approval in our country.

\section{Telemicrosurgery: the feasibility of robot-assisted microsurgery (Kiyohito Naito)}

Telemicrosurgery applications have expanded rapidly since the 1990s. The development of robotics has allowed a glimpse of new perspectives in nerve microsurgery. Minimally invasive surgery has rapidly become the first therapeutic option in many operative indications, reducing postoperative complications and increasing patient comfort. Sur-
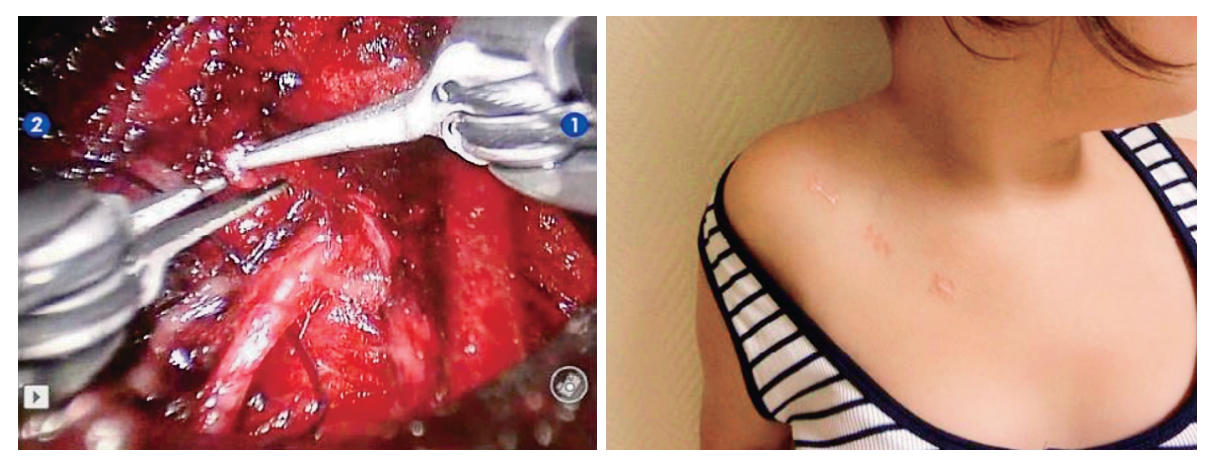

Figure-3 Telemicrosurgery of the brachial plexus surgery with da Vinci 
gical robotics has already proved useful, leading to numerous enhancements of the technique, especially in telemicrosurgery of the brachial plexus. In telemicrosurgery, more innovations should be developed through animal research and clinical study. However, we believe that the da Vinci system can further promote the application of telemicrosurgery. Its time has come!

\section{Robotic pediatric surgery (Hiroyuki Koga)}

Robotic surgery in children is an exciting new field with great potential. It involves the application of technology that minimizes stresses related to therapeutic intervention, which will greatly benefit pediatric patients requiring surgery. While it is only a matter of time before robotic surgery is routinely available for treating children, there are still technical issues related to the development of a robot dedicated to using the finer instruments required for pediatric surgical procedures. Equipment must also be adaptable to cater for a variety of sizes of patients including infants. So far, robotic instruments have been scaled down from $8 \mathrm{~mm}$ instruments used in adults to $5 \mathrm{~mm}$ instruments, and $2 \mathrm{D} / 3 \mathrm{D}$ telescopes have been developed. We anticipate that, as the number of complex pediatric laparoscopic/thoracoscopic procedures performed with robotic enhancement increases, there will be a measurable improvement in patient outcomes.

\section{The current state and future of robotic surgery in gynecology (Yasuhisa Terao)}

Robotic surgery has spread rapidly in the United States, Europe, South Korea, and elsewhere in recent years. Gynecological robotic surgery started in 2005 and is now performed more than in prostate surgery. Robotic surgery has been effective for delicate surgical operations in a relatively narrow surgical field, so gynecological pelvic surgery is a good adaptation. The benefits of robotic surgery include an increased range of motion with instrumentation, 3-dimensional stereoscopic visualization, and improved ergonomics for the surgeon. The greatest benefit is being able to reach a high technical level in a short period. Experience with laparoscopic surgery is advantageous when starting robotic surgery, as experts in laparotomy can shift to robotic surgery directly. Given the current state of robotic surgery in the United States and Europe, it should spread to gynecologic cancer surgery in Japan. Further improvements in its cost are necessary to establish standard robotic surgery that combines safety and comprehensiveness.

\section{Meeting summary and future directions (Atsuyuki Yamataka and Shigeo Horie)}

This symposium was held on September 11, 2014, on the occasion of the annual scientific meeting of Juntendo Graduate School of Medicine.

The advantages of robots over conventional laparoscopic surgery are clear and include: improved visualization, improved instrumentation that provides the surgeon with greater movement, better visualization and dissection, the ability to share and teach the procedures using a dual console, and a simulator. At the same time, there remain some obstacles to the introduction of robotic surgery. A complete portal approach in robotic surgery does not allow the surgeon to palpate the organ. To solve this problem, a life-sized 3D organ model will allow the surgeon to feel the outer surface of the organ. Furthermore, several instruments would facilitate the application of robotic surgery. A stapler, a vessel sealer, and a suction irrigator have been available for laparoscopic surgery, although wristed instruments at robotic arms that the surgeon can manipulate are more convenient and safe. These instruments are now on the horizon.

The future of robotic surgery is exciting. In terms of the further expansion of robotic surgical programs at Juntendo Hospital, safety is by far the most important issue. We have set up an ad hoc committee for robotic surgery at Juntendo Hospital to guide clinical trials and survey operative safety by peers. The surgical departments at Juntendo will move forward to new surgical approaches that are less invasive and more precise. 